\title{
Appunti per una storia pre-Leeuwenhoekiana degli «animalcula»
}

\author{
Di Luigi Belloni, Milano
}

Spicca, fra i documenti storici della biologia, la Lettera 18, in data 9 ottobre 1676, con cui Antoni van Leeuwenhoek (1632-1723) ${ }^{1}$ annunciò alla Royal Society di aver scoperto, in diverse acque, «animalcula» invisibili ad occhio nudo. Seguì, l'anno successivo, la non meno sensazionale notizia che anche il seme maschile pullula di «animalcula», essi pure osservabili soltanto con l'ausilio del microscopio. Spettacoli analoghi erano apparsi nel 1609/10 a Galileo Galilei (1564-1642) ${ }^{2}$, allorquando aveva elevato l'ingrandimento ottico a strumento di scienza: osservato attraverso il suo « occhiale», il cielo si era messo a «stellascere», a riempirsi cioè di stelle invisibili ad occhio nudo, e il chiarore diffuso delle nebulose e della via lattea si era risolto in una miriade di stelle ugualmente invisibili ad occhio nudo.

Prima del 1676, la microscopia aveva acuito ormai da tempo l'attenzione su altri «animalcula » - acari e anguillule - situati ai limiti della visibilità ad occhio nudo, che pullulano non soltanto in sostanze non viventi (formaggio, aceto), ma anche nello stesso organismo umano, quantunque limitatamente al mantello cutaneo. Ci possiamo quindi chiedere se il mondo degli scienziati - almeno dei c.d. «Neoterici» - non fosse ormai maturo per accogliere le mirabolanti scoperte del geniale «curioso della Natura». A questo problema intende contribuire, entro gli stretti limiti che le sono imposti, la presente nota, anticipando alcuni appunti dal materiale che vado raccogliendo da anni per una storia della microscopia nel Seicento ${ }^{3}$.

La validità dell'ingrandimento ottico e la sua potenzialità scientifica, affermate dal Galilei, trovarono entusiastici consensi in due opere capitali della innovazione scientifica: nel Novum organum (Londra 1620) di Francis Bacon (1561-1626) e nel Discours de la méthode (Leida 1637) di René Des Cartes (1596-1650). Nell'appendice di quest'ultimo dedicata a La dioptrique è inoltre raffigurato e descritto un microscopio semplice a lente

1 C. DobelL, Antony van Leeuwenhoek and his «little animals», Londra 1932, pp. 117-163.

2 Sidereus nuncius, Venezia 1610.

3 Per gli estremi bibliografici di altri nostri contributi, $c f$. L. Belloni, Zur Geschichte der tierforschenden Mikroskopie, in Nova Acta Leopoldina 30 (1965) nº 173, pp. 443-458. 
piano-convessa, che - per brevità di distanza focale, tipo di supporto e modalità di osservazione - sembra annunciare il microscopio Leeuwenhoekiano.

Svolta importante, nella evoluzione del microscopio semplice, fu l'impiego di una piccola sfera di vetro come mezzo diottrico: il c.d. microscopio «a pallina» o «a perlina», quale troviamo descritto e raffigurato nella Ars magna lucis et umbrae (Roma 1646) ${ }^{4}$ di Athanasius Kircher (1602-1680): «... alij novo fere, eoque sagacissimo invento, minimas sphaerulas vitreas, quarum diameter minimarum perlarum diametrum non excedit, huius videlicet quantitatis $O$, includunt tubulo cuidam: in huius superficie si pedem pulicis inter oculum, et lampadem posueris, videbis mirum dictu crus foemoris adinstar equini pedis horridum; pilus vero supra positus referet ingentem trabem, ut vel maxime mirum sit, quomodo in tam exigua sphaerula res tam immanes repraesentari possint».

Il Kircher racconta poi di aver ricevuto in dono cosiffatti microscopi dal cardinale (1644) Giovan Carlo DE' Medici (1611-1663), fratello del granduca Ferdinando II (1610-1670): e questa sua attestazione, che risale al 1646, è particolarmente significativa, sapendo che la carica di matematico granducale - già tenuta da Galileo - era allora ricoperta in Firenze da Evangelista Torricelli (1608-1647), cui viene per l'appunto attribuita l'invenzione del microscopio a perlina ${ }^{5}$. Il Torricelli morì a soli 39 anni nel 1647 , lasciando quale eloquente testimonianza dell'abilità non comune da lui raggiunta nel tagliare e polire lenti, la grande lente telescopica conservata al Museo di Storia della Scienza in Firenze, che, vagliata dall'ottica odierna, dimostra una «bontà eccezionale, paragonabile a quella che si può realizzare nelle migliori lenti lavorate coi metodi moderni più precisi $»^{6}$. Impari, se non altro, a tanta abilità appare quindi la ricetta ${ }^{7}$ usata dai suoi successori, che preparavano la perlina fondendo alla fiamma un filo di cristallo e la inserivano poi entro un supporto cartaceo.

Più progredito è pertanto il metodo esposto, sia pure succintamente, da Robert Hooke (1635-1703) nella Micrographia (Londra 1665) ${ }^{8}$, poiché esso comprende anche la politura della perlina e il montaggio entro un

4 pp. 834-835: «De mira rerum naturalium constitutione per Smicroscopium investiganda ».

5 Lezioni accademiche, Firenze 1715, pp. XVI-XIX. - Opere III, Faenza 1919, pp. 169 e 170 .

${ }^{6}$ V.RonchI, Evangelista Torricelli, ottico, in Convegno di studi Torricelliani, Faenza 1958, Faenza 1959, p. 117.

7 R. Caverni, Storia del metodo sperimentale in Italia I, Firenze 1891, pp. 508-509.

8 "The Preface», c. f. $1^{\mathrm{v}}$. 
supporto metallico: «and an Object, plac'd very near, be look'd at through it, it will both magnifie and make some Objects more distinct then any of the great Microscopes.» Benché otticamente superiori ai composti, i microscopi semplici sono, in compenso, «troublesome to be us'd, because of their smalness, and the nearness of the Object». Anzi, lo stesso Hooke ${ }^{9}$, avendo trovato "the use of them offensive to my eye, and to have much strained and weakened the sight ", finì per vedersi costretto a tralasciarne l'impiego, «though in truth they do make the object more clear and distinct, and magnifye as much as the double Microscopes; nay, to those whose eyes can well endure it, ' $t$ is possible with a single Microscope to make discoveries much better than with a double one, because the colours which do much disturb the clear vision in double Microscopes is clearly avoided and prevented in the single $»$.

Per comprendere la superiorità del microscopio semplice sul composto, così affermata da R. Hooke, bisogna tener presente che il microscopio composto - non ancora acromatizzato - produceva generosamente illusioni ottiche, e innanzitutto la c.d. immagine filamentoso-reticolare ${ }^{10}$, con la quale possiamo forse spiegare ${ }^{11}$ il meraviglioso brulichio di animaluzzi nei diversi parenchimi osservato da Theodor Kerckring (1639-1693) ${ }^{12}$ nel 1670: «Hoc quod instrumenti mei admirabilis ope clare detexi, visum est admirabilius: intestina scilicet, hepar, ceteraque viscerum parenchymata infinitis scatere minutissimis animalculis, quae an perpetuo suo motu ea corrumpant, an conservent, dubium esse posset ...» Duplice è l'interesse di questo brano del Kerckring:

$1^{\circ}$ perché attesta nel 1670 una recettività del mondo scientifico per l'esistenza di «minutissima animalcula» invisibili ad occhio nudo;

$2^{\circ}$ perché, dimostrando il vicolo cieco imboccato dal microscopio composto, mette indirettamente in risalto il valore dei perfezionamenti apportati dal Leeuwenhoek alla costruzione del microscopio semplice, da lui iniziata attorno al 1670. La minuscola lente a breve distanza focale tagliata e polita dal Leeuwenhoek con metodo mantenuto gelosamente segreto, conciliava infatti i due requisiti - ingrandimento e nettezza d'immagine (estesa

9 Microscopium 1679, p.312.

10 B.ZANobio, L'immagine filamentoso-reticolare nell'anatomia microscopica dal XVII al XIX secolo, in Physis 2 (1960) 299-317.

11 L.Belloni, Micrografia illusoria e «animalcula», in Physis 4 (1962) 65-73.

12 Spicilegium anatomicum, Amsterdam 1670, pp. 177-179: «Per Microscopia incertum in Anatomia judicium ». 
praticamente a tutto il campo $)^{13}$ - che gli permisero di scoprire il mondo degli esseri viventi invisibili ad occhio nudo.

Lo stesso Galilei non aveva tardato ad adattare il suo «occhiale» alla visione da-vicino e ad osservare con esso «minimorum animantium organa, motus et sensus ». Già nel 1610 ci è attestata la sua osservazione dell'occhio di «un certo insetto» - forse un ragno - in cui gli apparvero sette pertugi per la luce. A questa ed ad altre osservazioni entomologiche di Galileo seguirono quelle dei suoi colleghi dell'Accademia dei Lincei, che detengono, con la loro Melissographia (1625), il primato della iconografia biomicroscopica. Cosiffatte osservazioni microscopiche si limitavano alla superficie degli insetti; ma svelavano con tanta generosità strutture sconosciute e insospettate, che metteva conto di approfondire l'indagine entro il corpicino degli insetti, seguendo la via indicata da Giovanni Rucellai (14751525) nel poema Le api (Firenze 1539).

Io già mi posi a far di questi insetti

Incision, per molti membri loro,

(Che chiama anatomia la lingua greca),

racconta il Rucellai, prima di elencare, non senza stupore, i reperti da lui ottenuti sottoponendo all'ingrandimento di specchi concavi $i$ «membretti» dell'ape così preparati. L'esempio del Rucellai fu trascurato per un secolo: e purtuttavia esso insegnava ad accoppiare la microscopia con la dissezione dell'insetto, ossia con la «Microtome $»{ }_{1}^{14}$ per usare la terminologia di Marco Aurelio Severino (1580-1656) nella Zootomia Democritaea, pubblicata a Norimberga nel 1645. Nel precedente anno 1644 era apparso a Palermo L'occhio della mosca di G.B. Odierna (1597-1660), che, grazie alla finissima scomposizione praticata nell'occhio dell'insetto, precorre l'anatomia microscopica degli animali superiori. Questa verrà fondata da MArcello MALPIGHI (1628-1694) col De pulmonibus (Bologna 1661): lo stesso Malpighi che promuoverà, col De bombyce (Londra 1669), una nuova fase della entomotomia microscopica, legata particolarmente al nome di JAN SwAMmERDAM (1637-1680).

13 P. VAn der Star, Descriptive Catalogue of the simple microscopes in the Rijksmuseum voor de Geschiedenis der Natuurwetenschappen, Leyden 1953, p. 20.

14 p. 57. Frutto di «Microtome» è ad es. l'«uterus bicornis praelonga cervice, cum ovis et foetibus pusillis uniformibus» isolato da uno scarabeo e rappresentato nella minuscola figura a p. 346 . 


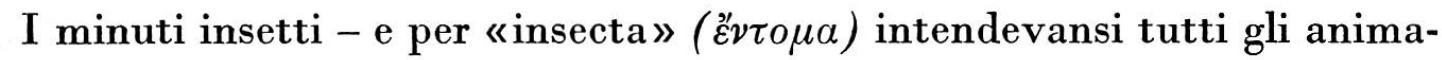
luzzi e, per antonomasia, quelli dal corpo «insectum» (strozzato, cioè, da intaccature o incisure) - funsero quindi da banco di prova al microscopio, il quale non mancò di ricambiare il servizio. Il microscopio, in altri termini, valorizzò gli insetti, svelando nei loro corpuscoli una organizzazione insospettata e differenziata in apparati. Lo studio microscopico dell'apparato ovoproduttore e dei caratteri specifici delle uova dei vari insetti sono punti importanti delle Esperienze intorno alla generazione degl'insetti (Firenze 1668) con cui Francesco Redi (1626-1697) negò la generazione spontanea o equivoca degli insetti, tradizionalmente ereditata da Aristotele.

Il microscopio fece inoltre cadere la concezione, essa pure tradizionale, che i più minuti insetti fossero molecole viventi indivisibili. Nella prefazione al De animalibus insectis (Bologna 1638) di Ulisse Aldrovandi (1522-1605) la piccolezza di certi insetti è a tal punto sottolineata «adeo ut $\stackrel{\alpha}{\tau} \tau \mu \alpha$ potius, quam $\ddot{\varepsilon}^{\prime} \nu \tau o \mu \alpha$ quaedam, quin pleraque recte dixeris ». Anche il termine «acaro », derivato dal greco, significa indivisibile.

Gli acari del formaggio, che ad occhio nudo sono "tanto minuti, che quasi paiono polvere ... veduti con quell'istrumento, diventano altrettanto grossi quanto le mosche senza ali, e si lasciano discernere tanto distintamente, che vi si riconoscono le gambe molto lunghe, la testa aguzzata e tutte le altre parti del corpo evidentissime $»{ }^{15}$ Cosiffatti reperti (1622) capovolgono la concezione dell'acaro: e il capovolgimento risalta ancor meglio nell'Insectorum, sive Minimorum Animalium Theatrum (Londra 1634), grazie al contrasto fra l'indivisibilità dell'acaro degli scabbiosi affermata da Thomas Moufet (1553-1604) ${ }^{16}$ e la strutturazione impartita allo stesso acaro da Théodore Turquet de MaYerne (1573-1655) mediante l'osservazione microscopica ${ }^{17}$.

Assicurata così alla scienza «mira corporum minutissimorum animalium constitutione, et fabrica», l'acaro è addirittura paragonato dal KIrcher $(1646)^{4}$ a un orso peloso. La struttura esterna dell'acaro del formaggio è

15 Lettera di N. Cl. Fabri de Pereisc (1580-1637) a Gerolamo Aleandro (1574-1629) in data 7 giugno 1622; cf. L. M. Rezzi, Sulla invenzione del microscopio, in Atti dell' Accademia Pontificia de' Nuovi Lincei 5 (1851) nota 19.

16 «Syronibus nulla expressa forma...praeterquam globi: vix oculis capitur magnitudo tam pusilla, ut non atomis constare ipsum, sed unum esse ex atomis Epicurus dixerit», p. 266.

17 «Imo ipsi Acari prae exiguitate indivisibiles, ex cuniculis prope aquae lacum quos foderunt in cute, acu extracti, et ungui impositi, caput rubrum, et pedes quibus gradiuntur, ad solem prodent» (nella «Epistola» dedicatoria). 
minutamente descritta nei suoi dettagli anche da Francesco Fontana (1590 ca.-1656) nelle Novae coelestium terrestriumque rerum observationes (Napoli 1646) ${ }^{18}$ e da Henry Power (1623-1668) nella Experimental Philosophy (Londra 1664) ${ }^{19}$. Il Power si sofferma anche ${ }^{20}$ sulla struttura esterna dell'acaro della scabbia con la sua «Appendent Proboscis or Trunk, consisting of many villous filaments in figure of a Cone, wherewith it perforates our skin, and sucks the blood or Aqueous nutriment from the pustules it is bred near». D'altra parte, R. Hooke, nella già ricordata Micrographia $(1665)^{21}$, descrive e raffigura accuratamente l'acaro reperibile in «all kinds of substances that are mouldy, or putrifying ", e insiste soprattutto sulle sue uova, ponendo così sul tappeto la validità della generazione spontanea per questi esseri viventi.

Da molecola vivente indivisibile, l'acaro è divenuto un «animalculum » con tanto di organizzazione differenziata in vari apparati, compresi quelli per l'aggressione e l'ovoproduzione: e questi reperti stanno alla base delle Osservazioni intorno a' pellicelli del corpo umano (Firenze 1687) - indirizzate al Redi - con le quali Giovan Cosimo Bonomo (1666-1696) dimostrò la etiologia acarica della scabbia, confortandola con la prova terapeutica, ossia coi risultati positivi ottenuti con medicamenti topici che "vagliano infallibilmente ad ammazzare $i$ pellicelli intanati anco nelle più riposte loro grotterelle e laberinti della cute». Anche se verrà inserita nella scienza ufficiale soltanto dopo un secolo e mezzo, questa scoperta del Bonomo inaugura la fase sperimentale della conquista del contagio vivo, e troverà il suo coronamento nell'opera del PASTE UR e del Koch, che il contagio vivo trasferiranno, per l'appunto, al mondo dei più minuti fra gli «animalcula» scoperti dal Leeuwenhoek.

Applicando medicamenti sulla cute degli scabbiosi, il Bonomo ne valutava l'azione acaricida con esperimenti in vivo. Un altro «animalculum»disponibile in colture spontanee su terreno liquido - si offerse invece per valutare in vitro l'attività di sostanze e di agenti fisici svariati.

«Quis credere posset acetum, et lac innumerabili multitudine vermium scatere, nisi id smicroscopa ars hisce ultimis temporibus summa omnium

18 Observatio $I$ : «Pulveris caseo innascentis », p. 148.

19 Observatio XII: «Mites in Cheese», pp. 16-17.

20 Observatio XIX: «Whey-worms, call'd by some, Wheal-worms, or Hand-worms, or Barrows », pp. 22-23.

21 Observatio LV: «Of Mites», pp. 213-215 e fig. 1 e 2 della tav. XXXVI. 
admiratione docuisset?", si chiede strabiliato A. Kircher $(1646)^{4}$ nell'inno entusiastico da lui elevato alle prime conquiste del microscopio nel mondo vivente. Alla valorizzazione degli «insetti» promossa dal microscopio, appartiene anche il rilancio dei vermicelli che Aristotele sembrava aver descritto nell'aceto quali precursori di insetti alati ${ }^{22}$.

I vermi dell'aceto appaiono meglio caratterizzati morfologicamente nelle Iatrologismorum seu medicinalium observationum pentecostae quinque (Roma $1652)^{23}$ del medico romano Domenico Panaroli (1587-1657): «sunt figurae oblongae, tenues, cum parvo capite, et tamquam anguillae conspiciuntur », come anguille, cioè, dotate di movimenti vivacissimi, che cessano con la morte, per cui esse sedimentano e formano una nubecola «quae multa mortuorum vermium cadavera putrefacta complectitur», ed è, al tempo stesso, generatrice di nuovi vermi.

L'acidità organolettica dell'aceto è proporzionale alla quantità dei vermi; ed è pertanto riferibile all'azione che questi esercitano sui recettori sensoriali, nei termini della rinnovata estesiologia meccanicista. La verminazione è più copiosa nell'aceto rosaceo che in aceti non aromatizzati; ed è anche in proporzione diretta alle dimensioni del recipiente.

La filtrazione, limpifica bensì l'aceto, ma non lo libera in permanenza dai vermi, poiché questi rinascono dopo tre giorni. Il vino aggiunto nella misura di 4-5 gocce a 1 libbra d'aceto, uccide completamente i vermi, che però rinascono, anche in questo caso, dopo tre giorni. Una «sterilizzazione » permanente si ottiene, invece, aggiungendo la triaca nella misura di 1 dramma ogni 2 libbre circa d'aceto: il vaso in cui tale aceto è rinchiuso va esposto al sole e agitato una volta al giorno: al termine di un mese, si lascia sedimentare la triaca e si decanta. L'aceto così ottenuto rimarrà sempre privo di vermi, anche senza venire esposto al sole: è l'«Acetum theriacale» da impiegare contro la peste e le febbri maligne: la triaca è l'antidoto vermicida. Ricordo, per inciso, che già nell' Ars magna il Kircher aveva segnalato i reperti «de sanguine febrientium verminoso», che svilupperà dodici anni più tardi nel celebre Scrutinium physico-medicum contagiosae luis, quae pestis dicitur (Roma 1658).

Nelle Noctes geniales (Bologna 1656) ${ }^{24}$, Giovanni Nardi (1585-1654), archiatra del granduca Ferdinando II di Toscana, si chiede come possa

${ }^{22}$ «Culices (conopes) autem proveniunt ex vermiculis, qui ex aceti faece oriuntur» (Hist. anim. $V$, cap. 20$)$.

${ }^{23}$ Observatio XXXVI: «Vermium in Aceto copia mirabilis, Microscopio visa », pp. 263-264.

24 «De nuper observatis Vermibus in Aceto», pp. 74-75. 
nascere putredine da una sostanza notoriamente antiputrida come l'aceto, e avendo visto verminare soltanto aceti derivati da vini svaniti e deboli («ex vappa, aut debilibus e vinis confecta»), ritiene che possa insorgere putredine "prae aqueo copioso, illoque impuro, superstite in Lora, et Vappa». L'insorgenza di putredine è invece esclusa per gli aceti derivanti da vino generoso, i quali rimangono per l'appunto immuni da anguillule.

La Observationum microcospicarum [!] centuria (L'Aja 1656) ${ }^{25}$ di Pierre Borel (1620 ca.-1689) si apre con l'osservazione dei «serpentes, seu anguillae, vel vermes serpentiformes, gressu sinuoso et satis celeri ambulantes» attraverso l'aceto. Oltre che «ope Perspicilli Microscopici», essi vermi «solo visu acri, ad solares radios cernuntur, in collo phialae aqua plenae», mentre nuotano verso l'alto in cerca d'aria («summitatem enim liquoris petunt, respirandi gratia»). Il Borel divulgò la conoscenza della verminazione acetica, provocando così in molte persone l'astensione dall'aceto. Alcunché di simile avverrà nell'estate del 1680 , quando «il étoit difficile de trouver dans Paris du vinaigre dans lequel il n'y eût point d'anguilles: et cela fit que bien des gens qui les avoient vûës dans nos Microscopes discontinuerent de manger de la salade $»{ }^{26}$ La conoscenza dei vermicelli dell'aceto era ormai divenuta di dominio comune: «adesso ogni uomo che sia di vista mediocre, li vede anco senz'occhiali», leggiamo in un interessante opuscolo propagandistico fiorentino ${ }^{27}$ del 1681.

Cladude Guillermet de Bérigard (1592 ca.-1663) inserì le anguillule dell'aceto nella seconda edizione del Circulus Pisanus (Padova 1661) ${ }^{28}$, e suggerì anche un metodo per facilitarne, si direbbe a occhio nudo, il ritrovamento.

Il rapporto quantitativo fra verminazione e acidità organolettica - ammesso, come si è visto, dal Panaroli - fu invece negato dal Power, che, nella Experimental Philosophy (1664) ${ }^{29}$, descrisse, egli pure, osservazioni ed esperimenti degni di particolare rilievo.

25 Observatio I: «De Vermibus aceti», pp. 7-8.

${ }^{26}$ L. Joвlot, Descriptions et usages de plusieurs nouveaux microscopes, tant simples que composez, Paris 1718, p. 2 e tav. I dell'appendice (Nouvelles observations).

27 Dichiarazione della vera causa de' bachi che si vedono nell' Aceto, e della morìa degli Animali. Con un racconto delle virtù, e nocumenti di esso Aceto. Considerazione Fisica, Naturale, ed Astrologica di Francesco Barzini Fiorentino professore di dette scienze, Firenze 1681.

28 «...ex innumeris vermiculis qui conspiciuntur ad Solem in scypho vitreo aceti pleno, praecipue cum linea visus ducitur a pede scyphi ad summama ceti superficiem» (p.535).

29 Observatio XXX: «Of the little white Eels or Snigs, in Vineger or Aleger», pp.32-36. 
Per esaminare in dettaglio questi che egli definisce «smallest of Animals», il Power porta l'indagine microscopica su gocce d'aceto deposte su di un vetro portaoggetti: le anguillule appaiono animate da movimenti vivacissimi verso la periferia, che si indeboliscono, e infine cessano, man mano procede l'essiccamento della goccia, permettendo così il rilievo dei caratteri morfologici dell'animaluzzo. Quanto alla osservazione ad occhio nudo, il Power suggerisce di introdurre una piccola quantità di aceto: $1^{\circ}$ in una fiala-da-essenze, cosicché appariranno sciami di anguillule «swimming at the edges of the Liquor »; $2^{\circ}$ «into a little cylinder of white glass, of a small bore and length, either sealed or closed up with cork and wax at the one end", ossia in un tubicino di vetro ${ }^{30}$ che ricorda quelli largamente usati dal Leeuwenhoek per studiare gli «animalcula» invisibili ad occhio nudo.

L'anguillula - che era destinata a divenire un animale prediletto per le ricerche sull'anabiosi - presenta, inoltre, il seguente comportamento di fronte ai più importanti agenti fisici e chimici:

a) è indebolita, come si è visto, dalla evaporazione dell'aceto, e infine uccisa, a disseccamento completo («after a little time dry quite away to nothing»);

b) è uccisa dal riscaldamento dell'aceto: e i cadaveri delle anguillule «stretched out at their full length» si accumulano sul fondo del vaso;

c) è analogamente uccisa dall'acido solforico, aggiunto nella misura di poche gocce a una fiala-da-essenze piena d'aceto;

d) sopporta, invece, il congelamento, poiché essa è in grado di sopravvivere per ore, ed anche per una notte, nell'aceto agghiacciato, e di riprendere rapidamente i movimenti quando l'aceto sia ritornato allo stato liquido. Se viene congelato aceto sovrastratificato d'olio, le anguillule abbandonano l'aceto e si rifugiano nell'olio; ma ritornano nel liquido nativo in seguito al riscaldamento.

Nella Micrographia (1665) ${ }^{31}$ R. Hooke prende atto degli esperimenti del Power, e si limita ad aggiungerne uno per dimostrare la stretta aerobiosi dell'anguillula: includendo l'aceto inverminato «in a small Viol, and stop'd very close from the ambient air, all the included Worms in a very short

30 Capovolgendo il tubicino, l'aceto rimane trattenuto nel lume ed è percorso da una bolla d'aria che si dirige verso l'alto. Quando essa ha raggiunto la sommità, succede talvolta di osservare alcune anguillule " on the top of it, crawling over the smooth convexity of the bubble (like so many Eels over a Looking-glass) without breaking thorow the tender cuticle and film of so brittle and thin a substance».

31 Observatio LVII: "Of the Eels in Vinegar», pp. 216-217 e fig. 3 della tav. XXV. 
time died, as if they had been stifled». Notevole, nella stessa opera, è poi l'iconografia delle anguillule osservate microscopicamente in campo oscuro: per analizzare il movimento vermicolare, R. Hooke pesca le anguillule « out of their Pond of Vinegar, by the net of a small piece of filtring Paper» e le depone su di un vetro nero e liscio, in modo «that they could wriggle and winde their body, as much almost as a Snake».

Nel 1669 J.SwammerdaM ${ }^{32}$ aggiunse all'acaro e all'anguillula un altro «animalculum »: la «pulce d'acqua», ossia la comune Daphnia. È questo, precisamente, l'«animalculum» usato dal Leeuwenhoek, all'inizio della Lettera 18, quale unità di misura cui riferire i primi «animalcula» da lui scoperti, che sono, per l'appunto, «more than ten thousand times smaller» rispetto alla Daphnia ${ }^{33}$. Nel contesto della stessa lettera, anche l'acaro del formaggio ${ }^{34}$ e l'anguillula dell'aceto ${ }^{35}$ diverranno «animalcula » giganteschi - se è permessa l'antitesi - e serviranno per dar miglior risalto alla nuova dimensione di vita scoperta dal Leeuwenhoek.

Nei 60 anni pre-Leeuwenhoekiani della microscopia (1610-1670), il microscopio semplice fu oggetto di perfezionamenti, finché, per merito precipuo del Leeuwenhoek, esso chiuse vittoriosamente la gara col microscopio composto, in attesa che questo potesse fruire di un obiettivo acromatico di impiego pratico.

Negli stessi anni, il microscopio acuì l'attenzione sugli «animalcula» situati ai limiti della visibilità ad occhio nudo, come le anguillule e gli acari, i quali ultimi, proprio per merito del microscopio, vennero trasformati da «atomi viventi» in organismi complessi.

Acari e anguillule schiusero alla scienza le meraviglie del mondo piccolo - sensibilizzando a questo uomini, come Hooke, che avrebbero avuto tanta parte nella accettazione delle scoperte del Leeuwenhoek - e furono le prime vittime dell'offensiva che portò l'uomo alla conquista del contagio vivo.

32 Historia Insectorum Generalis, ofte Algemeene Verhandeling van de Bloedeloose Dierkens, Utrecht 1669.

33 DobelL, l.c., p. 117.

34 Un altro «animalculum» scoperto dal Leeuwenhoek, pure dell'acqua piovana, appare tanto piccolo rispetto all'acaro del formaggio, «so is the size of a honey-bee to that of a horse" (DoBELL, l.c., p. 123).

35 Alla quale vengono riferiti dal Leeuwenhoek alcuni «animalcula» da lui scoperti nell'infuso di pepe: «As a worm of the bigness of a big pin, is to an eel of the thickness of one's wrist: So are these very little living creatures or eels in the pepper-water, to the size of the eels in vinegar» (DoBELL, l.c., p. 141). 